\title{
Features of perceived neighborhood environment associated with daily walking time or habitual exercise: differences across gender, age, and employment status in a community-dwelling population of Japan
}

\author{
Tzu-an Chen $\cdot$ Jung Su Lee $\cdot$ Kiyoshi Kawakubo \\ Etsuko Watanabe $\cdot$ Katsumi Mori • \\ Tadashi Kitaike $\cdot$ Akira Akabayashi
}

Received: 9 August 2012/ Accepted: 7 March 2013/Published online: 2 April 2013

(C) The Japanese Society for Hygiene 2013

\begin{abstract}
Objective Perceived neighborhood environment (NE) is considered a determinant of daily physical activity (PA). However, evidence concerning differences among types of PA, gender, and age is limited. The study purpose was to clarify the association between NE and walking time (WT) or habitual exercise (HE) across gender, age, and employment status in a community-dwelling population of Japan.

Methods A questionnaire mail survey with a stratified random 7,515 sampling was conducted in a northeast city in January 2007. Multiple logistic analysis was conducted to examine the associations between seven NE indices and WT or HE across gender, age, and employment status: 20-39 (young-employed), 40-59 (middle-employed), and 60-79 (old-employed or old-unemployed) after adjustment for age and means of transportation.

Results A total of 3,806 residents (52.4\% females) completed the survey. Traffic and crime safety in old-
\end{abstract}

T. Chen $\cdot$ J. S. Lee $(\varangle) \cdot$ E. Watanabe $\cdot$ K. Mori

Department of Health Promotion Science, School of Public

Health, Graduate School of Medicine, The University of Tokyo,

7-3-1 Hongo, Bunkyo-ku, Tokyo 113-0033, Japan

e-mail: jslee@m.u-tokyo.ac.jp

K. Kawakubo

Department of Food Sciences and Nutrition, Kyoritsu Women's University, Tokyo, Japan

T. Kitaike

Division of Health Science, Graduate School of Nursing,

Chiba University, Chiba, Japan

A. Akabayashi

Department of Biomedical Ethics, School of Public Health,

Graduate School of Medicine, The University of Tokyo,

Tokyo, Japan unemployed males and proximity to service facilities and traffic and crime safety in middle-employed females were significantly associated with a low risk of insufficient WT. Proximity to service facilities in old-employed males, number of service facilities, places for walking, and good view in middle-employed females, and density of dwelling and proximity to service facilities in old-unemployed females were significantly associated with a low risk of non-HE.

Conclusions The association between NE and WT or HE differed across type of PA, gender, age, and employment status, and was observed mainly in middle- and old-aged females. The middle- and old-aged female residents' PA possibly were more influenced by their NE, and NE would contribute to promote active living.

Keywords Age - Employment status - Gender .

Neighborhood environment · Walking · Habitual exercise

\section{Introduction}

Insufficient physical activity (PA) is the 4th leading risk factor for global mortality [1], and physically active living is widely recommended for preventing non-communicable diseases including coronary heart disease, type 2 diabetes, some cancers, clinical depression, and other chronic disorders [2]. The PA is any bodily movement produced by the skeletal muscles that uses energy. Moderate intensity PA includes walking or household chores, and vigorous intensity PA includes running, swimming or moving heavy loads. Current world public health recommendations emphasize the benefits of engaging in at least $150 \mathrm{~min}$ of moderate-intensity aerobic PA most days of the week, or at least 75 min of vigorous-intensity aerobic PA throughout 
the week [3]. However, it is estimated that $60-80 \%$ of the world's population does not meet the recommendations required to induce health benefits [4].

Among moderate-intensity PA, walking is the most popular daily PA [5]. However, the average number of walking steps has substantially decreased during the past 10 years particularly among the representative population of Japan [6]. Exercise is planned, structured, and repetitive PA for the purpose of conditioning parts of the body, thus exercise behavior is different from daily walking. The goal of the national public health recommendation for the Japanese adult is to increase daily walking steps by 1,500 , and to participate in aerobic exercise twice a week for a duration of $30 \mathrm{~min}$ on a regular basis [7]. Identifying determinants of walking and exercise behavior is important for increasing PA.

The perceived features of the neighborhood environment (NE) around the residence show a compelling association with residents' PA and have become a focus because the influence of the NE is larger at the population level and is expected to be sustainable [8]. The NE is now commonly used in studies of PA to refer to neighborhoods where the environment is more conducive to physically active [9]. In this idea, NE is focusing on the NE of the residences and does not include the location of the workplace where residents work in different regions. The NE includes residential density, accessibility to various destinations, street connectivity of sidewalk, walking infrastructure, aesthetics, and neighborhood safety [10, 11].

Evidence-based effective environmental intervention and policy approaches to promote PA were proposed and have already begun in Western countries: infrastructure initiatives through urban design of land use and planning on community and street scales and active transport policy and practices [12]. Furthermore, a guideline from the American Heart Association included environmental change as a population approach to enhance PA to prevent cardiovascular diseases [13]. The NE may vary largely from country to country depending on the cultural, social and physical context; however, studies concerning the influence of NE on PA in Japan are limited and have not yet been made clear.

Walking or exercise behaviors are different across gender and age groups [6] because social roles and the purpose of social interaction are different depending on gender and age groups. Thus, the association between perceived NE and walking or exercise behavior possibly varies according to gender and age groups. However, the majority of studies considered gender or age as covariates in statistical analysis, and a few studies focused on only gender difference [14-19], or only age-group difference [20], or specific gender or specific age group [21-23]. There is no published study that has revealed gender and age group differences in the association between perceived
NE and daily walking time (WT) or habitual exercise (HE) simultaneously in a population. Furthermore, the employment status may have significant influences on the association between NE and daily PA because the frequency of going out from the neighborhood to other regions as well as the duration of staying in the neighborhood might be different between employed and unemployed residents.

It is worthwhile to understand the gender, age, and employment status specific association between the features of perceived NE and WT or HE in a population in Japan.

The purposes of the study were (1) to clarify the association between the features of perceived NE and WT or $\mathrm{HE}$, and (2) to examine whether the association is different across gender, age, and employment status in Japan.

\section{Methods}

Study region and population survey

The study region was Tsuruoka City in Yamagata Prefecture in northeast Japan. According to the 2005 Census of Japan, the city has a medium-sized population of 142,384 in a $1,311-\mathrm{km}^{2}$ area. A population survey was conducted in January 2007. The survey was carried out in cooperation with the city to monitor the health status and health behaviors of the citizens. A sample of $7.0 \%$ of the residents was selected using a stratified random sampling method among residents aged 20-79 years old by gender and a 5-year age strata on the basis of data from the Basic Resident Register on March 31 in 2006. As a result, 7,515 residents were selected.

A cover letter and a self-administered anonymous questionnaire were directly sent to the selected residents by mail. Introduction of the study with the aims, procedure of the study assuring that the study was conducted anonymous and no individual would be identified in analyzing and reporting the data, and encouragement for participation were included in the cover letter. The questionnaire contained items about perceived NE, daily walking time, participation in exercise, and demographic variables. The demographic variables included gender, age, employment status, and main means of transportation.

When the questionnaire was returned, the resident was considered to have agreed to participate in the study. This study procedure was approved by the Ethical Committee of the Graduate School of Medicine, The University of Tokyo, Japan.

Perceived neighborhood environment

The Abbreviated version of the Neighborhood Environment Walkability Scale (ANEWS) was used to measure the 
residents' perception of NE $[24,25]$. In the ANEWS, NE is focusing on the influence of the NE on residents and does not include the location of the workplace.

Neighborhood was defined as the area within an approximately 10-min walking distance from a residence, and seven NE characteristics were assessed: density of dwelling (degree of density of residential buildings), proximity to service facilities (land use mix with nonresidential service facilities, such as restaurants, works and retail stores), number of service facilities within a 10-min walk (land use mix-access), street connectivity (intersection is short and easy to cross, and few dead end streets), places for walking (sidewalks on most of the streets and are separated from the traffic road), good view (trees along the streets or natural scenery while walking), and traffic and crime safety [17, 24, 25]. Reliability and internal consistency of the Japanese version of the ANEWS were tested and were shown to be moderate to high [17, 25].

Walking times and habitual exercise

Two types of daily PA with different intensity, WT and HE, were queried separately. Daily WT containing every kind of walking, e.g., commuting, shopping, working, or leisure in a whole day, was queried separately for average weekdays and for weekends. The sum of WT for weekdays and weekends was weighted as daily WT.

To test the validity and repeatability of the selfreported WT, a validation and repeatability study was conducted from September to November 2012. Study participants were recruited from residents who participated in a health promotion program managed by a ward health promotion center. Sixty-six residents recorded the daily number of steps using a pedometer for 11 days. When the diary of steps was reported, a questionnaire for the WT for weekdays and weekends was distributed twice at one-week intervals. As a result, 44 residents with range of age from 46 to 77 completed those three kinds of survey $(62.0 \pm 6.7$ years old, 12 males $)$. The testretest repeatability of weekly WT was relatively high (Pearson's product moment correlation coefficient $r=0.80)$. The external validity of weekly WT with the average of daily walking steps during 11 days was tested, and a difference of the mean of walking steps was 1,440 steps/day (7,769 steps/day and 9,208 steps/day) between the two groups divided by the median of weekly WT. Thus the validity of weekly WT was considered moderate and reasonable.

Participating in sports or exercise was also queried. If residents participated in sports or exercise all year round, the duration of each time and frequency per week were queried. The number of minutes of each time and frequency per week were used to define $\mathrm{HE}$ as doing exercise or sports equal to or more than $30 \mathrm{~min}$ at one time with a frequency of more than once a week [26].

Statistical analysis

Study residents were divided into six groups by gender and age: 20-39 years (young-aged), 40-59 years (middleaged), and 60-79 years (old-aged). Employment status was divided into currently employed (including self-employed or part-time job) or not. Main means of transportation was categorized as using an automobile or not.

To examine the association between NE and WT or HE by multiple logistic regression analysis, the scores of seven features of NE indices were converted into two categories using the median of each index from all respondents and used as independent variables. Insufficient WT or non-HE was used as a dependent variable. The median of daily WT was $60 \mathrm{~min} /$ day, thus daily WT was converted into two categories; insufficient WT ( $<60 \mathrm{~min} /$ day) or sufficient WT ( $\geqq 60 \mathrm{~min} /$ day). The HE was also divided into two categories; non-HE ( $<30 \mathrm{~min}$ at once or $<$ once a week) or HE ( $\geqq 30 \mathrm{~min}$ at once and $\geqq$ once per week during all year round).

Analysis was conducted according to the gender, age, and employment status, separately. However, the range of age in each group was 19 years, thus age was used as a covariate. Main means of transportation may have significant influence on daily PA and was included as a potential confounding factor. Adjusted odds ratios (OR) and $95 \%$ confidence intervals $(95 \% \mathrm{CI})$ were calculated. Equal to or more than the median score of each perception of $\mathrm{NE}$ index indicates that NE is conducive to PA compared to less than the median of each NE index (references), thus an OR less than 1.00 indicated low risk of insufficient WT or non-HE.

All statistical analysis was conducted using the SAS statistical package, Windows version (9.2 version; SAS Institute, Cary, NC, USA). All analyses were two-tailed, and a $p<0.05$ was considered statistically significant.

\section{Results}

A total number of 3,903 (51.9\%) residents responded to the survey. Valid information about gender, age, and WT was provided by 3,806 participants.

Table 1 showed the demographic characteristics according to gender and age groups. More than threefourths of young- and middle-aged males and females were employed, but three-fourths of old-aged females were not employed. Around $70 \%$ of all groups of males and females used automobiles, but less than half of old-aged females used automobiles as a main means of transportation. Irrespective of gender and age groups, around $60 \%$ did not 
Table 1 Study participants' characteristics according to gender and age groups

\begin{tabular}{|c|c|c|c|c|c|c|c|c|}
\hline & \multicolumn{4}{|l|}{ Male } & \multicolumn{4}{|l|}{ Female } \\
\hline & $\begin{array}{l}\text { All } \\
N=1,810\end{array}$ & $\begin{array}{l}20-39 \text { years } \\
N=284\end{array}$ & $\begin{array}{l}40-59 \text { years } \\
N=680\end{array}$ & $\begin{array}{l}60-79 \text { years } \\
N=846\end{array}$ & $\begin{array}{l}\text { All } \\
N=1,996\end{array}$ & $\begin{array}{l}20-39 \text { years } \\
N=349\end{array}$ & $\begin{array}{l}40-59 \text { years } \\
N=792\end{array}$ & $\begin{array}{l}60-79 \text { years } \\
N=855\end{array}$ \\
\hline Age (year) (mean $\pm \mathrm{SD})$ & $56.7 \pm 15.1$ & $31.4 \pm 5.6$ & $50.7 \pm 5.5$ & $70.0 \pm 5.6$ & $55.4 \pm 15.2$ & $31.2 \pm 5.4$ & $50.6 \pm 5.6$ & $69.6 \pm 5.6$ \\
\hline \multicolumn{9}{|l|}{ Employment status $^{\mathrm{a}}$} \\
\hline Employed (\%) & 71.4 & 86.9 & 91.7 & 49.1 & 57.1 & 81.6 & 79.2 & 26.4 \\
\hline \multicolumn{9}{|l|}{ Main means of commuting } \\
\hline Automobile users (\%) & 79.6 & 85.4 & 86.0 & 71.1 & 69.5 & 87.4 & 82.9 & 47.1 \\
\hline \multicolumn{9}{|l|}{ Habitual exercise ${ }^{b}$} \\
\hline $\begin{array}{l}\text { Non-habitual } \\
\text { exercise }(\%)\end{array}$ & 59.7 & 57.7 & 58.4 & 61.0 & 62.5 & 67.7 & 58.4 & 64.0 \\
\hline \multicolumn{9}{|l|}{ Walking time ${ }^{c}$} \\
\hline$<60 \mathrm{~min} /$ day $(\%)$ & 50.1 & 55.6 & 57.2 & 40.7 & 41.9 & 48.2 & 45.1 & 34.5 \\
\hline
\end{tabular}

Data are given as the mean \pm SD or percentage

${ }^{a}$ Employed: Agriculture, forestry, fishery, self-employed, full-time, and part-time job; unemployed: Housewife, student, and unemployed

b Doing exercise or sports equal to or more than $30 \mathrm{~min}$ at one time with a frequency of more than once a week all year round

${ }^{c}$ Daily walking time including every kind of walking, e.g., commuting, shopping, working or leisure in a whole day for average weekdays and for weekends

participate in HE. The prevalence of insufficient WT ranged from 34.5 to $57.2 \%$ and was lowest in old-aged males and females. Thus, the following analysis was restricted to young-aged and middle-aged employed, and old-aged employed and unemployed males and females, separately.

Table 2 showed the associations between perceived NE and insufficient WT according to gender, age, and employment status as the adjusted OR (95\%CI) after adjustment for age and means of commuting. Equal to or more than the median score of each perception of NE index was categorized as high. Thus, a high score of each NE index indicates that NE is conducive to PA.

In the male residents, a high score of traffic and crime safety was significantly associated with a low risk of insufficient WT in the old-aged unemployed group. There were no significant associations between perceived NE and WT in the young- and middle-aged groups.

In the female residents, a high score of proximity to service facilities and a high score of traffic and crime safety were significantly associated with a low risk of insufficient WT in the middle-aged employed group. There were no significant associations between perceived NE and WT in the young- and old-aged groups.

Density of dwelling, number of service facilities within $10 \mathrm{~min}$, street connectivity, places for walking, and good view were not significantly associated with WT in any ages of males and females.

Associations between perceived NE and non-HE according to gender, age, and employment status were shown in Table 3 as the adjusted OR (95\% CI) after adjustment for age and means of commuting.
In the male residents, only a high score of proximity to service facilities was significantly associated with a low risk of non-HE in the old-aged employed group, and there were no significant associations between perceived NE and $\mathrm{HE}$ in young- and middle-aged groups.

In the female residents, a high score of number of service facilities within $10 \mathrm{~min}$, a high score of places for walking, and a high score of good view were significantly associated with a low risk of non-HE in the middle-aged employed group, and a high score of density of dwelling and a high score of proximity to service facilities were significantly associated with a low risk of non-HE in the old-aged unemployed group. There were no significant associations between perceived NE and HE in young- and old-aged employed groups.

Street connectivity and traffic and crime safety were not significantly associated with HE in any ages of males and females.

\section{Discussion}

The study results revealed that all features of perceived NE except street connectivity were associated with daily PA. However, the association differed not only across gender, age, and employment status but also the type of PA in a community dwelling population after adjustment for age and automobile commuting. The association between NE and PA was observed mainly in middle- and old-aged females.

The current study results support the previous reports that females were more likely to walk for transportation or 


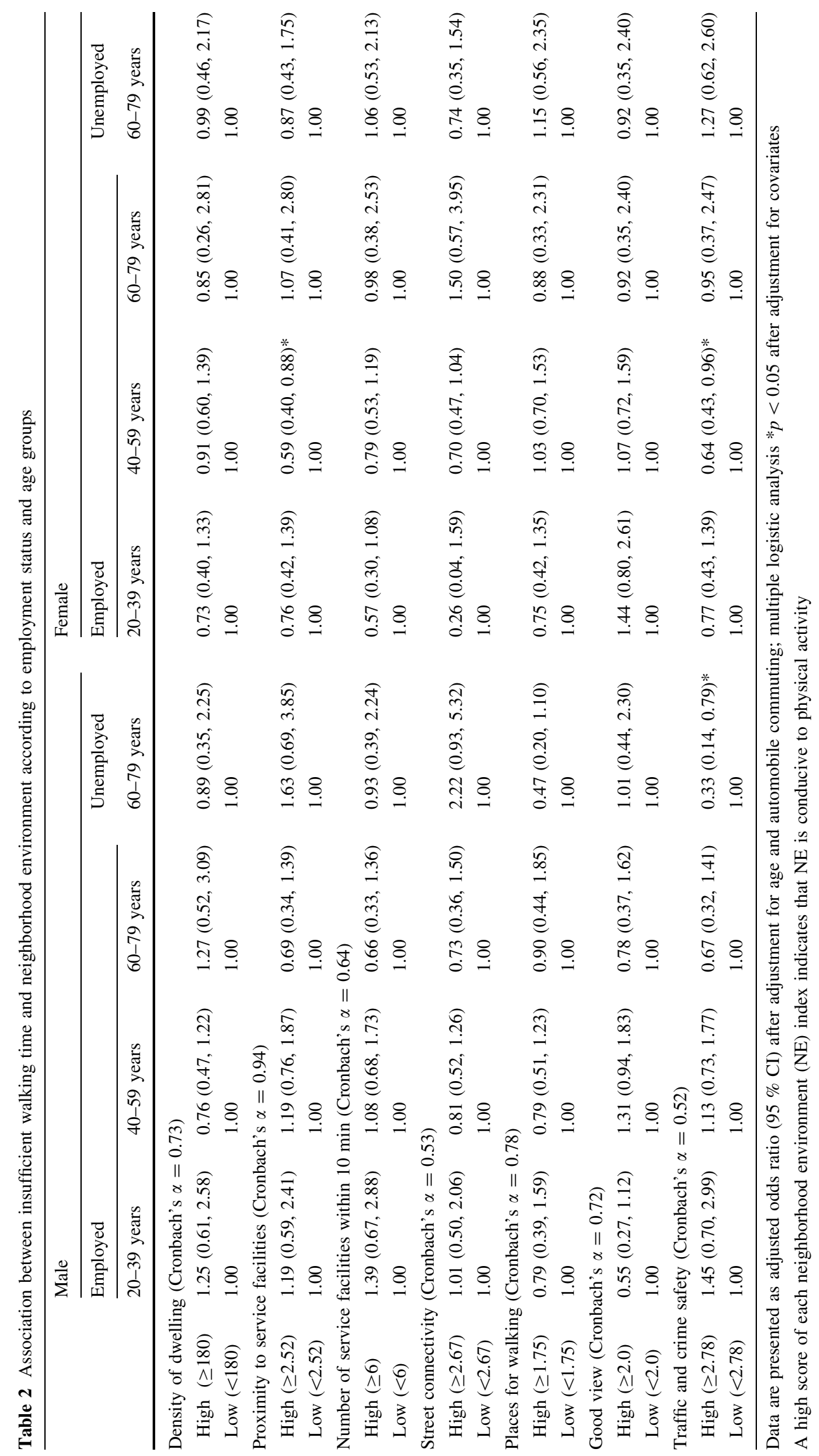




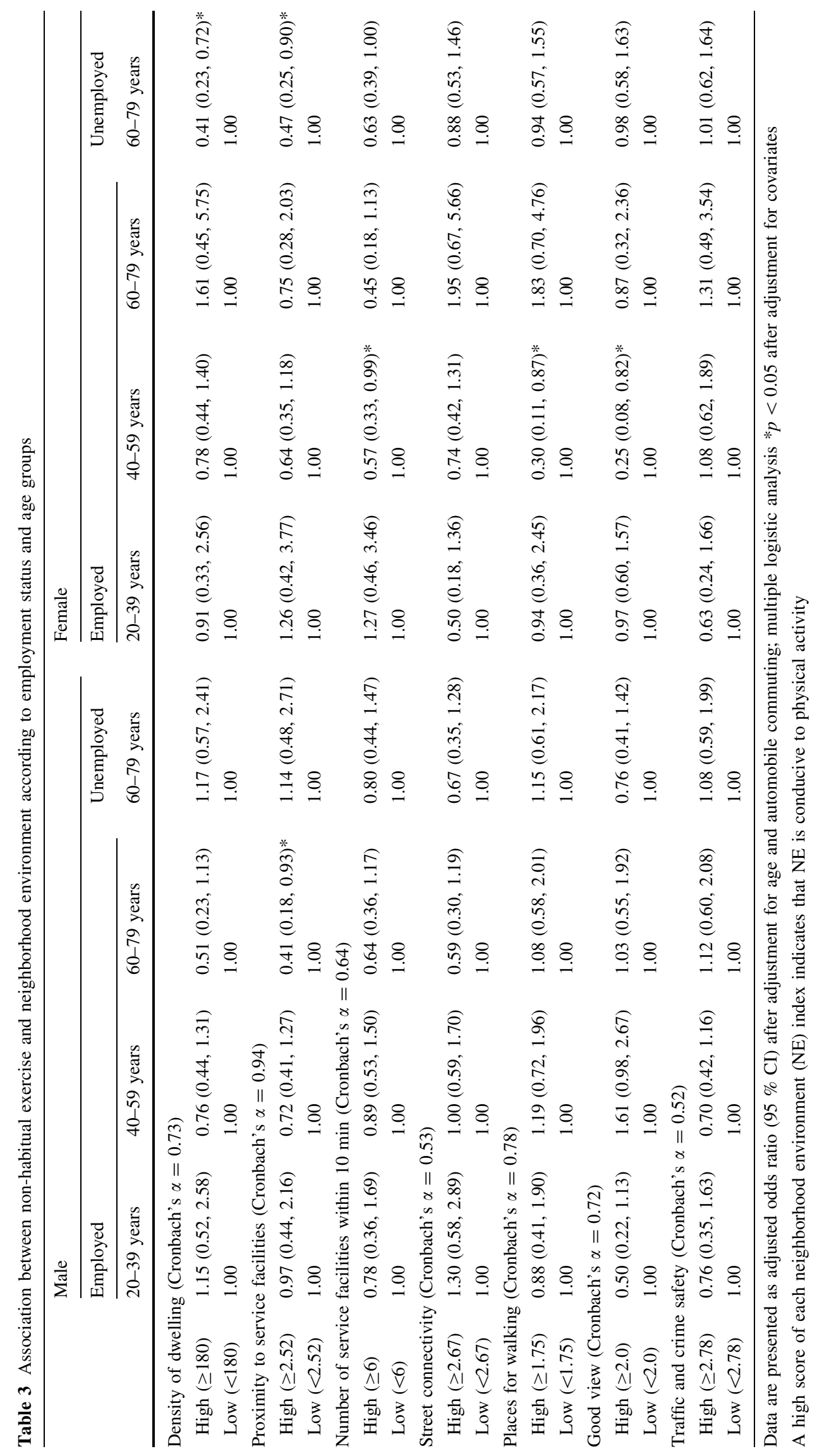


walk for exercise in their neighborhood [27, 28], and middle- and old-aged females might spend longer time in their neighborhood compared to young-aged females or males and might be more influenced by their NE. The current study results suggest that NE would contribute to promote active living particularly for female residents.

Although increasing studies have focused on the associations between features of NE and PA, the results have been inconsistent because of regional differences, gender and age dependent differences, and/or types of PA for specific purposes. A review study summarized that those living in a residentially dense area showed longer WT [11]. In the current study, perception of a high score of density of dwelling was associated with a low risk of non-HE for old-aged unemployed females. This result might suggest that old-aged females living in a residentially dense area tend to walk for exercise, and such a NE might indicate an ease of transport to exercise and/or sports facilities.

The perception of a high score of proximity to service facilities was associated with a low risk of insufficient WT in middle-aged employed females and with a low risk of non-HE in old-aged employed males and old-aged unemployed females. Also, a high score of number of service facilities within 10 min was associated with a low risk of non-HE in middle-aged employed females. Several studies showed that proximity and accessibility to destinations such as shops and service facilities especially influence transport walking $[20,29]$. Accessibility to various destinations has been reported to be associated with longer WT $[10,11]$ and many studies reported gender difference in the association $[14,16,19,21,27,28]$. Accessibility to abundant destinations would be important for females because of their social role in daily house work, including daily shopping, especially in Japan.

Furthermore, the perception of a high score of places for walking was associated with a low risk of non-HE in middle-aged employed females. The availability of recreational resources in the neighborhood, i.e., community center, gymnasium, and park, consistently showed to be one of NE that influences exercise behaviors [30, 31]. The proximity and accessibility of many kinds of service facilities within walking distance and places for walking in the neighborhood for regular exercise could enhance daily PA particularly in old-aged males and middle- and oldaged females.

The perception of a high score for good view indicates that there are interesting things to look at while walking, trees along the streets, or natural scenery and was associated with a low risk of non-HE for middle-aged employed females. Good view may be an important feature of walking for exercise or walking for transportation to exercise and/or sport facilities particularly for the females. To understand these kinds of gender and age differences from the influences of NE, further study including objectively measured NE will be needed.

The perception of a high score for safety was correlated with the low risk of insufficient WT in old-aged unemployed males and middle-aged employed females but did not associate with HE. In the current study, safety contained aspects both of traffic and crime, and the score of safety (median 2.78) was the highest among seven NE indices. The proportion of insufficient WT was lowest in old-aged males among the males, thus unemployed oldaged males and employed middle-aged females were more likely to walk for transportation in their neighborhood when they perceived their neighborhood is highly safe from traffic and crime.

There are several limitations in this study. First, questions regarding WT did not distinguish purpose-specific walking, e.g., for transportation or for leisure. Previous studies reported that street connectivity was related to walking for transportation while a good view was related to walking for exercise [17]. However, because of the difficulty in distinguishing purpose-specific WT in daily life, WT, which included all kinds of walking such as commuting, shopping, and leisure, was queried to help induce more accurate recall. Secondly, a previous study pointed out the mismatch of subjective and objective measurements of NE [32]. Further study using objective measurements of NE or WT will be needed. Thirdly, among the seven indices of NE, street connectivity did not show significant association with either WT and HE for any ages of males and females in this study. Street connectivity indicates the short distance between intersections, fewer dead ends, and better connective routes. A study conducted in another region in Japan reported that street connectivity was associated with WT [16]. The Cronbach's $\alpha$ of the street connectivity in the current study, which indicate internal consistency of each item of questionnaire, was relatively low. This may be why the association could not be observed in the current study. Further, Cronbach's $\alpha$ of the traffic and crime safety was also relatively low possibly because two different aspects of safety were included, and the association between NE and HE could not be observed any ages of males and females. Further study will be needed to improve the Japanese version of NE scale.

The strength of this study was that it was conducted in a region with a sizable population and clarified the differences of association between features of NE and WT or HE across gender, age, and employment status for the first time in Japan.

Although many environmental intervention studies and urban planning policies have been conducted for implementation of health behavior to prevent non-communicable disease such as cardiovascular disease and diabetes mellitus in Western countries [12,33], there are comparatively 
few studies concerning association of NE and PA in Japan. Thus, awareness about the influence of NE on healthrelated behavior is scarce until now. Environmental and policy approaches, e.g., creation and improvement of access to places for PA, community- and street-scale urban design and land use, active transport policy and practices, and community-wide policies and planning, are recommended based on scientific evidence [13]. To determine the priority of NE according to scientific evidence in Japan, further studies will be required. Lastly, implementing the policy and intervention on urban planning will be expected in the near future if evidence is accumulated depending on the social and cultural context of Japan.

\section{Conclusion}

The association between perceived NE and daily PA was different not only across gender, age, and employment status but also the type of PA, and the associations were mainly observed in middle- and old-aged females regardless of age and commuting means. The middle- and old-aged female residents' daily PA possibly were more influenced by their NE, thus, features of NE would contribute to promote particularly females' active living. The local infrastructure including places for walking, safety and accessibility to destinations should be designed to ensure facilitating walking for transportation or for exercise.

Acknowledgments The authors would like to express their sincere gratitude to the municipal officials and residents who participated for their cooperation. This study was partly supported by a grant from the Japan Ministry of Education, Culture, Sports, Science and Technology.

\section{References}

1. World Health Organization (WHO). Global health risks. Mortality and burden of disease attributable to selected major risks, 2009. http://www.who.int/healthinfo/global_burden_disease/Global HealthRisks_report_full.pdf. Accessed 5 Dec 2012.

2. World Health Organization (WHO). Monitoring framework and targets for the prevention and control of NCDs, 2012. http:// www.who.int/nmh/events/2012/consultation_april_2012/en/index. html. Accessed 5 Dec 2012.

3. World Health Organization (WHO). Global recommendations on physical activity for health. 2010. http://whqlibdoc.who.int/ publications/2010/9789241599979_eng.pdf. Accessed 5 Dec 2012.

4. World Health Organization. Global health observatory. Prevalence of insufficient physical activity. Geneva; 2008. http://www. who.int/gho/ncd/risk_factors/physical_activity/en/. Accessed 5 Dec 2012.

5. Siegel PZ, Brackbill RM, Heath GW. The epidemiology of walking for exercise: implications for promoting activity among sedentary groups. Am J Public Health. 1995;85:706-10.
6. Ministry of Health, Labour and Welfare. 2009 National Health and Nutrition Survey. Tokyo; 2011. http://www.mhlw.go.jp/ toukei/itiran/gaiyo/k-eisei.html\#kokumineiyou. Accessed 5 Dec 2012.

7. Ministry of Health, Labour and Welfare. Healthy Japan 21, The second plan. 2012. http://www.mhlw.go.jp/bunya/kenkou/ kenkounippon21.html. (in Japanese). Accessed 5 Dec 2012.

8. Marcus BH, Williams DM, Dubbert PM, Sallis JF, King AC, Yancey AK, et al. What we know and what we need to know: a scientific statement from the American Heart Association Council on Nutrition, Physical Activity, and Metabolism (Subcommittee on Physical Activity); Council on Cardiovascular Disease in the Young; and the Interdisciplinary Working Group on Quality of Care and Outcomes Research. Circulation. 2006;114:2739-52.

9. Rydin Y, Bleahu A, Davies M, Dávila JD, Friel S, Grandis GD, et al. Shaping cities for health: complexity and the planning of urban environments in the 21st century. Lancet. 2012;379: 2078-9.

10. Owen N, Humpel N, Leslie E, Bauman A, Sallis JF. Understanding environmental influences on walking; Review and research agenda. Am J Prev Med. 2004;27:67-76.

11. Saelens BE, Handy SL. Built environment correlates of walking: a review. Med Sci Sports Exerc. 2008;40(7 Suppl):S550-66.

12. Heath GW, Parra DC, Sarmiento OL, Andersen LB, Owen N, Goenka S, for the Lancet Physical Activity Series Working Group, et al. Evidence-based intervention in physical activity: lessons from around the world. Lancet. 2012;380:272-81.

13. American Heart Association. AHA scientific statement: Population approaches to improve diet, physical activity, and smoking habits. Circulation 2012. http://circ.ahajournals.org/content/ early/2012/08/19/CIR.0b013e318260a20b. Accessed 5 Dec 2012.

14. Humpel N, Owen N, Iverson D, Leslie E, Bauman A. Perceived environment attributes, residential location, and walking for particular purposes. Am J Prev Med. 2004;26:119-25.

15. Inoue S, Murase N, Shimomitsu T, Ohya Y, Odagiri Y, Takamiya $\mathrm{T}$, et al. Association of physical activity and neighborhood environment among Japanese adults. Prev Med. 2009;48:321-5.

16. Inoue S, Ohya Y, Odagiri Y, Takamiya T, Ishii K, Kitabayashi M, et al. Association between perceived neighborhood environment and walking among adults in 4 cities in Japan. J Epidemiol. 2010;20:277-86.

17. Kondo K, Lee JS, Kawakubo K, Kataoka Y, Asami Y, Mori K, et al. Association between daily physical activity and neighborhood environments. Environ Health Prev Med. 2009;14: 196-206.

18. Lee JS, Kawakubo K, Kohri S, Tsujii H, Mori K, Akabayashi A. Association between residents' perception of the neighborhood's environments and walking time in objectively different regions. Environ Health Prev Med. 2007;12:3-10.

19. Lee JS, Kondo K, Kawakubo K, Kataoka Y, Mori K, Umezaki M, et al. Neighborhood environment associated with daily physical activity measured both objectively and subjectively among residents in a community in Japan. Jpn J Health Human Ecol. 2011;77:94-107.

20. Shigematsu R, Sallis JF, Conway TL, Saelens BE, Frank LD, Cain KL, et al. Age differences in the relation of perceived neighborhood environment to walking. Med Sci Sports Exerc. 2009;41:314-21.

21. Inoue S, Ohya Y, Odagiri Y, Takamiya T, Kamada M, Okada S, et al. Perceived neighborhood environment and walking for specific purposes among elderly Japanese. J Epidemiol. 2011; 21:481-90.

22. King WC, Belle SH, Brach JS, Simkin-Silverman LR, Soska T, Kriska AM. Objective measures of neighborhood environment and physical activity in older females. Am J Prev Med. 2005; 28:461-9. 
23. Li F, Fisher KJ, Brownson RC, Bosworth M. Multilevel modelling of built environment characteristics related to neighbourhood walking activity in older adults. J Epidemiol Community Health. 2005;59:558-64.

24. Cerin E, Saelens BE, Sallis JF, Frank LD. Neighborhood Environment Walkability Scale: validity and development of a short form. Med Sci Sports Exerc. 2006;38:1682-91.

25. Inoue S, Ohya Y, Odagiri Y, Takamiya T, Ishii K, Lee JS, et al. Reliability of the abbreviated neighborhood environment walkability scale Japanese version. Jpn J Phys Fitness Sports Med 2009;58:453-462. (in Japanese).

26. Ministry of Health, Labour and Welfare. Healthy Japan 21: The National Health Movement in the 21st Century. Tokyo; 2000. (in Japanese).

27. Stronegger WJ, Titze S, Oja P. Perceived characteristics of the neighborhood and its association with physical activity behavior and self-rated health. Health Place. 2010;16:736-43.

28. Suminski RR, Poston WS, Petosa RL, Stevens E, Katzenmoyer LM. Features of the neighborhood environment and walking by U.S. adults. Am J Prev Med. 2005;28:149-55.
29. Frank LD, Pivo G. Impacts of mixed use and density on utilization of three modes of travel: single-occupant, transit, and walking. Transp Res Rec. 1994;1466:44-52.

30. Diez Roux AV, Evenson KR, McGinn AP, Brown DG, Moore L, Brines S, et al. Availability of recreational resources and physical activity in adults. Am J Public Health 2007;97:493-499.

31. Sallis JF, Hovell MF, Hofstetter CR, Elder JP, Hackley M, Caspersen CJ. Distance between homes and exercise facilities related to frequency of exercise among San Diego residents. Public Health Rep. 1990;105:179-85.

32. Gebel K, Bauman AE, Sugiyama T, Owen N. Mismatch between perceived and objectively assessed neighborhood walkability attributes: prospective relationships with walking and weight gain. Health Place. 2011;17:519-24.

33. Lee IM, Shiroma EJ, Puska P, Balir SN, Katzmarzyk PT. Effect of physical inactivity on major non-communicable diseases worldwide: an analysis of burden of disease and life expectancy. Lancet. 2012;380:219-29. 